\title{
TRABAJAR EN EL CAMPO, VIVIR EN LA CIUDAD CONFORMACIÓN DE TERRITORIOS PERIURBANOS EN MISIONES
}

\section{Guillermo Neiman y Alfonsina Verónica Albertí}

\begin{abstract}
Resumen
La creciente residencia urbana de trabajadores agrícolas es una constante de las últimas décadas, asociada a la búsqueda de oportunidades laborales y al acceso a servicios y a políticas públicas de alcance limitado en el medio rural. La reducción y creciente estacionalidad de la demanda de trabajo en las plantaciones de yerba mate de Misiones (Argentina) llevó a la relocalización de trabajadores rurales temporarios en ciudades cercanas. Este artículo se orienta a comprender el barrio como un territorio que organiza las relaciones de trabajo y de reproducción social; se consideran las condiciones y prácticas de acceso al trabajo, las redes sociales y económicas, su significado como nuevo ámbito de socialización y las políticas públicas clave.
\end{abstract}

Palabras clave: agricultura, cosecheros, relocalización, ciudad, Argentina.

\begin{abstract}
Working in the fields, living in the city. Conformation of peri-urban territories in Misiones

The growing urban residence of agricultural workers has become a constant during the last decades, associated with the search for job opportunities, and the access to social services and to public policies usually limited in rural areas. The decline and growing seasonality of labor demand in the local herbs or yerba mate plantations in Misiones (Argentina) led to the relocation of temporary rural workers in nearby cities. This article seeks to understand the neighborhood as a territory that organizes labor relations and social reproduction, for which are considered the conditions and practices of access to work, the social and economic networks, its meaning as a new socialization milieu and the key public policies.
\end{abstract}

Keywords: agriculture, harvesters, relocation, city, Argentina.

Guillermo Neiman: Sociólogo. Investigador principal en el Centro de Estudios e Investigaciones Laborales, del Consejo Nacional de Investigaciones Científicas y Técnicas [CEIL-CONICET). Profesor titular de la Facultad de Ciencias Sociales, Universidad de Buenos Aires. Director académico de la Maestría en Estudios Sociales Agrarios de FLACSO Argentina.

ORCID iD: 0000-0002-8724-6454

Email: gneiman@ceil-conicet.gov.ar

Alfonsina Verónica Albertí: Antropóloga. Investigadora asistente en el CEIL-CONICET y docente auxiliar en la Facultad de Psicología (Universidad Nacional de La Plata).

ORCID iD: 0000-0003-2002-5275

Email: alfonbert@gmail.com

Recibido: 10 de marzo de 2021.

Aprobado: $?$ de mayo de 2021. 


\section{Introducción}

Los desplazamientos definitivos hacia centros urbanos por parte de asalariados agrarios se han intensificado en las últimas décadas en distintas regiones de América Latina. Esto ocurre en el marco de los procesos de restructuración productiva que atraviesa la agricultura y de las nuevas dinámicas de los mercados de trabajo, incidiendo finalmente en la configuración de los trabajadores y sus hogares.

Los nuevos territorios periurbanos se construyen sobre la base de la persistencia de movilidades urbano-rurales vinculadas al trabajo agrícola temporario y a la consolidación de agentes y mecanismos de contratación que sostiene esas movilidades, junto con la búsqueda e inserción en tareas no agrícolas. Además, se vuelve significativa la existencia de redes y dispositivos para apuntalar la reproducción social de estos grupos más allá de la centralidad del trabajo en este proceso, incluyendo las políticas sociales, las relaciones sociales derivadas del parentesco, la vecindad, la amistad y la solidaridad, que pasan a conformar un conjunto relativamente generalizado y articulado de acciones y recursos dirigidos a ese fin.

Este artículo aborda el fenómeno de la relocalización de trabajadores agrícolas para el caso de barrios periurbanos de la ciudad de Oberá y pequeñas localidades cercanas, núcleo de la producción e industrialización de la yerba mate en la provincia de Misiones, localizada en la región noreste de la Argentina. A partir de la década del noventa, una sucesión de cambios tecnológicos y de organización de esa producción llevó a una reducción y mayor concentración en el tiempo de la demanda de trabajo, incrementando la desocupación en una parte significativa de la población trabajadora rural y dando lugar al inicio de un proceso de migración y radicación en ciudades cercanas.

El propósito principal de este artículo es comprender el barrio como un territorio que organiza las relaciones de trabajo y reproducción de los trabajadores y sus familias. En este sentido, los principales interrogantes que orientan el análisis son los siguientes: ¿Cuáles son los motivos que promovieron esta relocalización? ¿Qué estrategias desplegaron los trabajadores y sus hogares para apropiarse de los territorios periurbanos? ¿Cómo se expresan las dinámicas laborales en el contexto de la apropiación de los territorios urbanos? ¿Qué implicancias tiene la presencia de una dinámica multiterri- 
torial para la reproducción social actual y futura de esta población según género y generaciones?

Para el año 2010, la ciudad de Oberá contaba con 63.960 habitantes (las estimaciones actuales indican 80.000 habitantes), lo que la convertía en la segunda ciudad de la provincia por su tamaño, con un incremento de aproximadamente un $50 \%$ con respecto a 2001 . También para aquella fecha, el departamento totalizaba 106.812 personas, de las cuales el 30,6\% correspondía a población rural localizada mayoritariamente en forma dispersa; además, entre las dos fechas, la población rural agrupada prácticamente se duplicó y la rural dispersa disminuyó un 30\% (INDEC, 2001, 2010).

En primer lugar, abordamos los procesos de construcción de estos territorios laborales y de reproducción social considerando perspectivas complementarias que nos permitan dar cuenta de la complejidad del caso analizado. Luego, realizamos una presentación empírica del caso basada en los cambios productivos y laborales de la producción de yerba mate, para, finalmente, comprender desde la perspectiva de los trabajadores el sentido que adquieren estos nuevos espacios y las potenciales implicancias de este cambio en sus trayectorias de vida.

El trabajo de campo se llevó a cabo durante el mes de octubre del año 2017; se realizaron 28 entrevistas a residentes en barrios periurbanos de Oberá y en pequeñas localidades circundantes. En todos los casos se trata de asentamientos conformados por cosecheros y excosecheros de yerba mate, entre los cuales, buscando abordar la heterogeneidad de situaciones y trayectorias, se entrevistaron personas según género y estratos de edad. Mediante las entrevistas, se reconstruyeron los motivos que llevaron a su relocalización, las estrategias laborales actuales de los sujetos, así como las formas en que estas se articulan con las estrategias más amplias de reproducción social de las familias y con las prácticas y relaciones que constituyen las redes vecinales y otras. A partir de las entrevistas, se destaca el vínculo que estos sujetos entablan no solo con el mercado laboral, sino también con el Estado a través de diversas políticas públicas a las que tienen posibilidades de acceso.

\section{Hacia una conceptualización de la relocalización urbana de trabajadores agrícolas}

La profundización y restructuración del desarrollo capitalista en la agricultura latinoamericana y la mayor flexibilización en la contratación de trabajadores llevaron a que la temporalidad, la transitoriedad y la eventualidad reemplazaran crecientemente a la estacionalidad clásica del empleo agrario (Lara, 1998; Piñeiro, 1999; Neiman y Quaranta, 2000; Barrón, 1999; Tubío, 2001). En este contexto, se viene identificando la relocalización de la mano de obra agrícola en localidades urbanas, principalmente como parte de las 
transformaciones del empleo en el sector (Graziano da Silva, 1982; Gómez y Klein, 1993). ${ }^{1}$

La movilidad hacia centros urbanos se explica por la presencia de períodos más prolongados de desocupación o inactividad rural, junto con la necesidad de insertarse en otras actividades, tanto por parte de los propios trabajadores como de otros integrantes de sus hogares. Así, los barrios o asentamientos periurbanos que se conforman condensan lo que Narotzky (2004) denomina "reproducción ampliada", a partir de la articulación entre el impulso de la población por lograr su subsistencia - lo que clásicamente se denomina reproducción-y el impulso de acumulación propio del capitalismo.

Los procesos de territorialización en espacios urbanos por parte de trabajadores agrícolas pueden expresarse en las estrategias sociolaborales que despliegan para lograr su reproducción social, por el efecto de la mayor cercanía física a los lugares de trabajo y a los agentes de contratación (Radonich, 2001), para la realización de múltiples intercambios económicos y culturales locales o comunitarios (Moreno, 2019), la circulación más o menos permanente entre áreas rurales y localidades urbanas (Crovetto, 2011) o por cierta autonomía que pueden alcanzar en estos lugares, a diferencia de las restricciones que en ese sentido impone la localización rural (Trpin, 2020). Para los casos de población involucrada en movimientos migratorios, estos espacios pueden oficiar como un eslabón para sostener la movilidad espacial, circulando a través de una amplia diversidad de territorios (Lara, 2012).

Entonces, el proceso de construcción de una nueva "territorialidad vinculante" (Pérez Martínez, 2016) implica una interacción permanente a nivel de las actividades productivas (agrícolas y no agrícolas) y de la movilidad de los trabajadores (rural y urbana). Así, una nueva geografía del trabajo agrario se corresponde con una renovada relación entre capital y trabajo, reconfigurando las condiciones de inserción laboral de la población y colocándola, a su vez, en un nuevo contexto de sociabilidad y de trabajo.

Cada vez con mayor frecuencia han ido considerando estos espacios urbanos como fuente de otros recursos, además de los exclusivamente laborales, para sostener la reproducción de estos grupos, estando entre los más referenciados el acceso a la vivienda, la educación y las políticas públicas focalizadas en estos sectores (Albertí, 2018; Albertí y Mingo, 2019).

De esta manera, se plantea la necesidad de ampliar el enfoque considerando no solo la relación del trabajo con el proceso productivo, e incluso con el mercado de trabajo, para incorporar la esfera del hogar y de los vínculos sociales que se establecen, sin centrarse tampoco exclusivamente en los comportamientos de los individuos trabajadores. Lo que se busca, entonces, es

1 Para la Argentina, hacia principios de este siglo, el $40 \%$ de los asalariados agrarios tenía residencia urbana, de los cuales la mitad se localizaba en ciudades cuya población era superior a los 10.000 habitantes (Quaranta, 2010). 
"resituarlo en una visión que parta de la complejidad y, en consecuencia, que incorpore el cruce, la articulación y confrontación de distintas dimensiones de la vida social en torno al trabajo" (Lindón, 2002b).

Así, el territorio emerge como una realidad creada a partir de la apropiación instrumental y simbólica de un espacio concreto (Raffestin, citado en Saquet, 2015). En este sentido, territorialidad y territorio, en tanto herramientas conceptuales, buscan comprender cómo se produce esa apropiación y cómo se organizan las prácticas y representaciones de los grupos sociales involucrados.

De lo que se trata es de la gestación de procesos más o menos simultáneos y asociados de desterritorialización y reconstrucción de una nueva territorialidad (Haesbaert, 2009). Para los trabajadores precarizados, ver reducidas sus posibilidades de inserción laboral implica la pérdida de control de un territorio, para iniciar, a través de su desplazamiento, un proceso de apropiación de nuevos espacios y, con ello, la expectativa de construcción de nuevas prácticas y trayectorias individuales y sociales. En tanto el espacio es constituyente de las relaciones sociales, no puede concebirse por fuera de ellas; necesariamente la reproducción social implica la producción de territorios; "[...] mientras que los grupos subalternos producen territorialización mediante apropiación del espacio los grupos hegemónicos lo hacen mediante la dominación" (Haesbaert, 2013, p. 19).

El proceso de habitar el territorio, entonces, conduce a su construcción a partir de la producción cotidiana que llevan adelante los sujetos, transformándolo en su nuevo espacio de vida a partir de sus propias prácticas y experiencias, dándole una densidad distinta al fenómeno de la urbanización de los trabajadores y sus familias (Lindón, 2015).

Una sociología del trabajo situada en el territorio permite abordar el trabajo realmente existente en un espacio geográfico y social determinado, a la vez que visibilizar las complejas experiencias de reproducción social de poblaciones vulnerables. Específicamente, coloca la dimensión laboral como resultado de una imbricación de prácticas concretas que tienen lugar a distintos niveles - individual, familiar, comunitario- y de una pluralidad de agentes privados y públicos que intervienen en la construcción de un determinado mercado de trabajo, en cuya conformación participa una diversidad de condicionantes (económicos, sociales, afectivos y políticos).

En el caso que analizamos en este artículo no se concreta un proceso de separación absoluta entre ambos tipos de territorios (rurales y periurbanos), sino que la necesidad de trabajar por parte de los cosechadores de yerba implica la implementación de prácticas que tienen en la pluriterritorialidad el eje organizador de la vida laboral. Esto deriva en "un saber circular" (Tarrius, 2000), que supone una socialización de los sujetos, en donde la movilidad espacial puede concebirse como un recurso esencial para la subsistencia, que implica un conocimiento del espacio y de las dinámicas de las redes sociales que lo atraviesan. 
Las interacciones entre los ámbitos doméstico, vecinal o comunitario y público-estatal (local o municipal, provincial e incluso nacional) y de estos con el trabajo - definido a partir del modelo productivo y de las características del mercado de trabajo - condicionan la relación trabajo/residencia para el caso de las áreas urbanas conformadas a partir de la crisis laboral en actividades agrícolas.

En los barrios tareferos ${ }^{2}$ de la ciudad de Oberá el mundo del trabajo agrícola se entremezcla con la vida cotidiana, es un espacio de socialización en donde se transmiten los saberes, la información y las estrategias necesarias para ser trabajador agrícola en estas circunstancias. En este artículo, concebimos al barrio cómo un dispositivo espacial que organiza y otorga sentido a la reproducción social de los trabajadores.

\section{Restructuración, trabajo y movilidad poblacional en la actividad yerbatera}

La provincia de Misiones es la mayor zona productora de yerba mate de la Argentina, en la que el gran crecimiento de la actividad se produjo durante la década del veinte del siglo pasado, en el marco de un intenso proceso de asignación y colonización de tierras fiscales con la obligación de plantar yerba (Bolsi, 1982; Slutzky, 2011; Gallero, 2019). De esta manera se consolidó el fin del período de tipo extractivo basado en plantaciones naturales disponibles en territorios de Brasil, Paraguay y Argentina. Además, específicamente para el caso argentino, marcó el inicio de una etapa de autoabastecimiento, ya que hasta entonces el consumo era mayoritariamente satisfecho desde Brasil y Paraguay.

La génesis del cultivo de yerba mate en la provincia se remonta hacia el siglo XIX, a partir del complejo proceso histórico organizado con la sedentarización de la población guaraní y su explotación laboral, primero mediante el sistema de encomienda y luego a través del sistema del "peonaje por deudas", que implicaba la retención de la mano de obra en los lugares de trabajo. $\mathrm{Al}$ endeudamiento atribuido con o sin base real seguía el reclutamiento compulsivo mediante "enganchadores", bajo amenazas de prisión; de este modo las empresas extractivas de yerbales naturales creaban sistemas privados de control, castigos y vigilancias al margen del sistema político formal (Rau, 2014a, 2014b).

La transición de yerbales naturales a cultivados, el poblamiento por medio de corrientes migratorias, principalmente brasileñas y europeas, y la colonización en pequeñas extensiones de tierra sentaron las bases de la nueva economía yerbatera en la provincia, algunas de cuyas características se extienden hasta la actualidad.

2 El uso de la denominación tareferos se extendió a partir de la prohibición de la actividad extractiva en los yerbales naturales (desde los años treinta) y junto al avance del poblamiento provincial por parte de migrantes de origen europeo. 
Hacia 1940 la superficie plantada alcanzaba las 60.000 hectáreas (en 1920 eran aproximadamente 5.000), la producción pasó de alrededor de 3 millones de kilos a los 100 millones en 1937, para luego estabilizarse en 70 millones por año. A su vez, por efecto de los procesos de colonización, la superficie promedio por explotación pasó de 21 hectáreas en 1928 a 8 hectáreas en 1935 (Bolsi, 1982). ${ }^{3}$

Simultáneamente, la población total del territorio misionero se incrementó de unas 63.000 personas en 1920 a 246.000 para el año 1947, con un $81 \%$ de habitantes rurales, mientras que el promedio nacional era $38 \%$ para esa misma fecha.

El departamento y la ciudad de Oberá (ver Mapa 1) se constituyeron rápidamente como el espacio más representativo del desarrollo agrícola y yerbatero de la provincia, conformándose en "el corazón del modelo misionero de la época, donde centenares de pequeñas y complejas unidades económicas, la típica chacra misionera, definían su rasgo principal" (Bolsi, 1982, p. 56). La imagen de una ruralidad provincial polarizada entre colonos de cierta prosperidad de origen europeo (Bartolomé, 1975) y trabajadores pobres -incluyendo paraguayos, brasileños y argentinos que circulaban por el territorio - se debía completar con agricultores de subsistencia, trabajadores de la agroindustria y pobladores de pequeñas localidades rurales proveedoras de servicios (Forni, 1965, p. 2). Un estudio pionero sobre cosecheros de yerba mate o tareferos en la provincia de Misiones (Flood et al., 1972) constata la percepción de bajos ingresos (que podían incluir remuneraciones no monetarias), una marcada inestabilidad laboral y deficientes condiciones de trabajo, todo ello agravado por malas situaciones habitacionales y bajos niveles de escolaridad.

Las recurrentes crisis económicas van a constituirse en un rasgo típico de la trayectoria productiva de la yerba mate en el país. Las repetidas situaciones de sobreoferta de producción — que al comienzo se explicaba por la disponibilidad de producción local e importada desde Brasil-, las variaciones en el consumo y la caída de precios se conjugaron para provocar la salida de pequeños productores, desocupación, empobrecimiento y migraciones rural-urbanas (Rofman, 2001).

3 El ciclo productivo de la yerba mate se inicia efectivamente al tercer o cuarto año de plantación y alcanza su máximo rendimiento entre los ocho y doce años, pudiendo estar en producción durante treinta años o más. 


\section{Mapa 1. Provincia de Misiones, departamento y ciudad de Oberá}

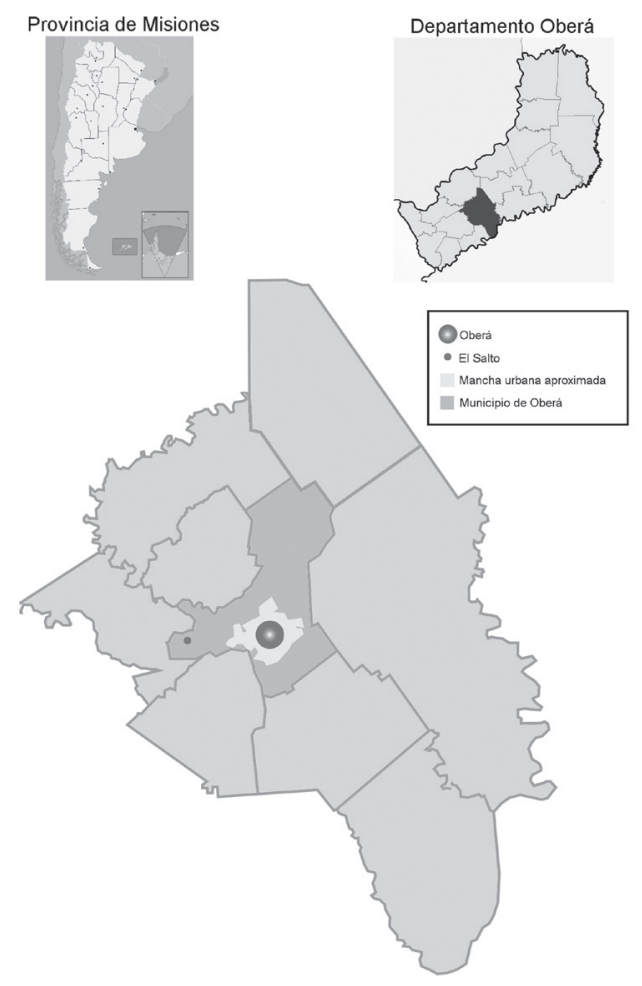

Fuente: Wikipedia, 200?.

Los primeros intentos por controlar esa situación a través del establecimiento de cupos de producción y cosecha con el objetivo de estabilizar los precios derivaron en la creación, en 1935, de la Comisión Reguladora de la Producción y Comercio de la Yerba Mate (CRYM). Estuvo en funcionamiento hasta el año 1991, cuando se desreguló totalmente la actividad en el marco de las políticas neoliberales que se implementaron en la Argentina y afectaron a numerosas producciones regionales.

Para esa década, el sector de la yerba mate atravesaba una desestructuración sin precedentes a partir de un incremento sustancial de la superficie plantada (cerca de un $40 \%$ ) y de la producción ( $80 \%$ ), la aparición de plantaciones con mayores extensiones y rendimientos por hectárea, conformándose un modelo de desarrollo profundamente desigual (Slutzky, 2011; Gómez Lende, 2016). A fines de los años noventa, el precio de la yerba equivalía a un $10 \%$ del promedio de los primeros cinco años de la misma década (Gorenstein, Schorr y Soler, 2011), el movimiento cooperativo que nucleaba a colonos se debilitaba notablemente (Rodríguez, 2018), el segmento agroin- 
dustrial se concentraba y se reducía a la mitad la cantidad de secaderos y molinos yerbateros (AACREA, 2003).

En 2002, se creó el Instituto Nacional de la Yerba Mate (INYM), una entidad pública no estatal cuya primera función es arbitrar en la fijación de los precios para mejorar la situación de los productores. Se inició un proceso de expansión de la actividad en el que la superficie plantada se incrementó un 12,6\% entre 2002 y 2011 (Gortari, 2016), en el marco de una tendencia fuertemente concentradora de la economía yerbatera (Gortari y Sena, 2018) y una creciente subordinación de los pequeños productores a los complejos agroindustriales (Schiavoni, 2008).

Según el Censo Nacional Agropecuario de 2002, de las 27.955 explotaciones agropecuarias registradas en la provincia el $60 \%$ declaraba plantaciones de yerba y el $80 \%$ de estas tenía menos de 10 hectáreas plantadas. ${ }^{4}$ Para esa misma fecha el departamento Oberá poseía un $15 \%$ de la superficie total plantada de la provincia (aproximadamente 22.000 hectáreas) y un $30 \%$ del total de "hoja verde" procesada, constituyendo la principal jurisdicción en cuanto a tierra plantada y producción.

Hacia finales de la primera década de este siglo, en la zona centro de la provincia (a la que pertenece el departamento Oberá), un 35\% de la superficie con yerba estaba ocupada por pequeños productores, con una densidad de 1000 plantas por hectárea, un rendimiento medio de 35 quintales por hectárea y una participación del $20 \%$ de la producción total; por su parte, los productores grandes alcanzaban rendimientos de 80 quintales por hectárea, duplicaban la densidad de plantación y explicaban la mitad de la producción de yerba del departamento. En la etapa industrial, los 10 molinos más grandes (de algo más de 100 existentes en la provincia) concentraban el $75 \%$ de la comercialización mayorista de la yerba (Colcombet, 2010).

Se estima que alrededor de 15.000 personas se ocupan del corte de la yerba (Sena, 2017), aunque algunos cálculos alcanzan los 20.000 trabajadores (Rau, 2014a). ${ }^{5}$ La Encuesta de Asalariados Agrarios (Ministerio de Trabajo, Empleo y Seguridad Social de la Nación, 2014) contabilizó para la provincia un total de 21.458 asalariados agrarios, ${ }^{6}$ de los cuales algo menos de un cuarto estaba empleado en forma permanente y los temporarios estaban

4 Según el Censo Nacional Agropecuario de 2018, hay 23.341 explotaciones agropecuarias en la provincia (aún no está disponible el dato por departamento), con una disminución de un $15 \%$ respecto al relevamiento anterior, equivalente a prácticamente la mitad de la caída registrada a nivel nacional.

5 En cuanto al trabajo asalariado permanente en todas las explotaciones agropecuarias de la provincia, el Censo Nacional Agropecuario de 2002 contabilizó 6.698 ocupados de esa condición; los resultados preliminares del Censo Nacional Agropecuario de 2018 llegan a las 3.690 personas.

6 La definición de asalariado agrario para esta encuesta corresponde a las personas ocupadas al menos durante 30 días continuos o discontinuos durante los 12 meses previos a su aplicación. 
ocupados 6,6 meses al año en promedio; según esta misma fuente, solo un $23,8 \%$ de ese total registraba contribuciones a la seguridad social. Además, para 2002, un total de 7.999 explotaciones agropecuarias contrató trabajadores temporarios para la "cosecha de otros cultivos" (excepto la producción de granos), de las cuales el $70 \%$ lo hizo por medio de contratistas y otras formas de tercerización laboral (INDEC, 2002).

En un contexto de relativa estabilidad en lo que hace a los volúmenes y composición del empleo durante las últimas décadas, se destacan, sin embargo, transformaciones a nivel de la organización de la producción de yerba mate y el funcionamiento de los mercados de trabajo propiamente dichos, lo cual termina impactando en los perfiles de los trabajadores.

En primer lugar, considerando el ciclo anual de trabajo, la demanda laboral se "estacionaliza" aún más como consecuencia de los mayores requerimientos relativos para las tareas de cosecha (Fabio, 2009). Esto sucede por un doble fenómeno que se observa entre las empresas medianas y grandes: por un lado, el aumento de la mecanización y el uso de insumos químicos para el laboreo de los cultivos, que lleva a una reducción de los requerimientos laborales durante la precosecha. Por ejemplo, la aplicación de herbicidas desciende de 15 horas/hectárea a 3 horas/hectárea, según se realice en forma manual o mecanizada.

Por otro lado, los mayores rendimientos por hectárea asociados principalmente al aumento en la densidad de las plantaciones por hectárea, provoca que el pico de trabajo de la cosecha se haga más pronunciado aún (el período de zafra se extiende entre los meses de marzo y septiembre, si bien los días efectivos de trabajo difícilmente exceden los 120). ${ }^{7}$ Así, para las explotaciones de hasta 2500 plantas por hectárea, con fertilización y aplicación de herbicidas, los requerimientos de cosecha pueden oscilar de 60 a 90 horas por hectárea, dependiendo de la zona productora, descendiendo significativamente hasta entre 10 y 20 horas por hectárea para pequeños productores con menos de 1000 plantas por hectárea (Colcombet, 2010). ${ }^{8}$

Por otro lado, la creciente difusión de la subcontratación laboral y el aumento en la residencia urbana de los tareferos y sus hogares constituyen una marca definitoria de los cambios en el mercado de trabajo de la yerba desde los años noventa hasta la actualidad.

La predominante contratación indirecta o tercerizada se concreta por medio de contratistas cuyo crecimiento se hizo notorio con la desregulación

7 Durante los meses de noviembre y diciembre se lleva a cabo la zafriña, con aproximadamente 30 días de trabajo efectivo.

8 Las modalidades mecánicas o semimecánicas de cosecha no tienen todavía una difusión importante, particularmente entre las empresas yerbateras de la provincia de Misiones. La incorporación de guinches, carritos de transporte y tijeras automáticas, entre los más importantes, son cambios que han tenido más aceptación y afectan la productividad del trabajo, aunque su aplicación dependerá de las características de las plantaciones y de las decisiones de los productores. 
de la actividad en los años noventa (Rau, 2014b). Incluso ha sido frecuente, al menos en los primeros años de expansión de este fenómeno, que fueran las propias empresas agroindustriales las proveedoras de un capital inicial o algún apoyo administrativo para promover el desarrollo de la intermediación (Ibarguren, 2017).

Queda bajo la responsabilidad de estos intermediarios la movilización, el transporte, la organización y la administración de los cosecheros; la necesidad de movilizar grandes contingentes de trabajadores y la posibilidad de constituir sociedades con un muy bajo nivel de formalización promueven la difusión de la intermediación (Fabio, 2009). A su vez, el incumplimiento de los crecientes requisitos de calidad de la materia prima lleva a sancionar a los contratistas, quienes presionan sobre los capataces y estos sobre los trabajadores. ${ }^{9}$

Por último, el desplazamiento de tareferos hacia ciudades intermedias se asocia a la crisis de la producción familiar y campesina que se intensificó desde los años noventa, la generación de una sobreoferta de mano de obra originada en la mencionada restructuración de la producción, la ampliación de oportunidades laborales en los centros urbanos y, además, la posibilidad de acceso a la asistencia estatal (subsidios, vivienda) y a otros servicios.

Un relevamiento de tareferos realizado en barrios periféricos de la ciudad de Oberá muestra que la mitad de esos trabajadores eran originarios de la misma ciudad y el resto se dividía entre los provenientes de localidades y poblados rurales cercanos y también de ciudades más lejanas (Aparicio, Re y Pereyra, 2016). A su vez, de los no nacidos en Oberá prácticamente la mitad se había desplazado durante las décadas de 1990 y 2000 (y otro 25\% lo había hecho en los años ochenta).

\section{Prácticas y perspectivas de los trabajadores sobre la vida en los barrios}

\section{"Antes había más trabajo, mientras que el patrón no te echaba vos tenías laburo"}

La creciente falta de trabajo en la agricultura aparece como la causa principal, aunque no necesariamente la única, de la movilidad de la población rural hacia Oberá. El escenario más generalizado que explica esa tendencia se relaciona con las transformaciones en la producción, que pueden incluir cambios tecnológicos y comportamientos de los empleadores, que terminan afectando prácticamente por igual a trabajadores con y sin tierra, a trabajadores jóvenes y más experimentados, a los varones y a las mujeres que par-

9 Al igual que para otras producciones y para la misma época, la intermediación laboral intervino sobre las necesidades de las empresas de reclutar trabajadores en grandes volúmenes y para momentos críticos y puntuales, transferir la organización y el control del proceso de trabajo y establecer el vínculo legal y administrativo con los trabajadores (Neiman, 2010). 
ticipaban de las tareas, a los que se ocupaban exclusivamente en la yerba y a los que también vieron disminuidas las posibilidades de insertarse en tareas para otros cultivos.

La imagen de un campo con "más trabajo", propia de la etapa anterior, significaba básicamente mayores posibilidades de estar ocupado durante todo el año - aun cuando fuera en condiciones precarias e inestables-, completando la tarefa con otras tareas durante el resto del ciclo productivo de la yerba. En la secuencia de cambios posteriores a esa época, los trabajadores hacen referencia a la incorporación de tecnologías que llevaron a la desaparición de ciertas tareas que regularmente se llevaban a cabo en los períodos entre cosechas. Para Ramón (36 años, barrio La Cantera), "antes había algo más de laburo, algún productor te daba alguna carpida, además se cortaba la yerba con machete, se picaba [...]", argumento que completa Luis (35 años, Los Helechos), al sostener que

con el paso del tiempo fueron como poniendo más maquinaria y descartaron más los trabajos de mano de obra, después ya no querían más las mujeres y los chicos por la ley, ${ }^{10} \mathrm{y}$ después descartaron a los hombres también.

La tarefa era la ocupación principal, por los ingresos que generaba para los trabajadores y sus hogares y por el tiempo que insumía a lo largo del año; sin embargo, las demás tareas eran las que les garantizaban sobrevivir durante períodos de ingresos escasos pero que mantenían cierta regularidad y cuando los consumos se restringían a un mínimo. Estas tareas también correspondían a la yerba, pero podían complementarse con otros cultivos -el té, por ejemplo, que comenzó a atravesar transformaciones similares-, junto a otras changas ocasionales de menor relevancia. Aun cuando la identificación con la tarefa era determinante, la ruralidad ofrecía otras opciones para apuntalar la reproducción.

La referencia a los patrones en la etapa previa a la migración incluye no solo la correspondiente a su función de empleador, sino también como alguien más cercano a la vida cotidiana y a las necesidades de los trabajadores. En este sentido, los cambios exceden las experiencias de trabajo propiamente dicho, para extenderse hasta el desplazamiento de los patrones del medio rural como responsables de gestionar personalmente el trabajo en sus propias explotaciones agrícolas. La salida del campo de los patrones, aunque sin perder su vínculo económico y las modalidades de contratación de los trabajadores basadas en intermediarios, completa el escenario de la ruptura de una época con "más trabajo".

10 Se refiere a los mayores controles que se fueron ejerciendo sobre las condiciones de contratación, formalización y transporte de los trabajadores, que se intensificaron desde principios de este siglo, principalmente sobre el trabajo de las familias y, en especial, de los hijos menores de edad. 
Carlos (53 años, Los Helechos) sostiene:

Hay mucho cambio, antes los patrones tenían sus casas en la chacra, tenían su personal temporario, no temporario, que era un peón general que tenía para toda la campaña, mientras que el patrón no te echaba vos tenías laburo. Hoy por hoy ni un patrón tiene una casa en la chacra porque no quiere tener responsabilidad del peón... ahí faltó un poco de exigencia, de responsabilidad del patrón, de velar por ese personal, tenerlo, cuidarlo y darle trabajo. Hoy por hoy hay patrones que no quieren ser responsable(s) de un personal que está trabajando en la chacra, entonces ellos quieren tratar de buscar un empleador, un contratista que le(s) haga el trabajo.

El otro sostén de las economías domésticas rurales — que también entró en crisis - era la autoproducción de alimentos y la eventual venta o intercambio de algunos excedentes, práctica que se desarrollaba en tierra propia o en parcelas pertenecientes al empleador que podían utilizarse para realizar alguna plantación, aunque mayormente ese uso terminaba constituyéndose en una forma de pago por la ejecución de ciertas tareas (excepto la cosecha). Justamente, las visiones más clásicas vincularon la relocalización de trabajadores agrícolas en espacios periurbanos con la descomposición de las economías campesinas; sin embargo, en el caso de los tareferos este tipo de situación no es la única, sino que forma parte de una amplia diversidad de escenarios.

Por ejemplo, Carlos (53 años, Los Helechos), que trabajó y residió en varias chacras de patrones para quienes trabajaba como cosechero de yerba mate, a los 40 años decidió migrar para ocuparse en la construcción en Buenos Aires; a los 48 años regresó y se instaló en un barrio de Oberá por motivos familiares (cuidar al padre de 90 años, con problemas de salud). Cuando vivía en zonas rurales, los patrones le "prestaban" una porción de terreno para que produjera alimentos para la familia:

Nosotros vivíamos en la chacra del patrón, de ahí te daba la tierra para que vos limpies y que vos plantes. Entonces entre medio de esa plantación o sea le plantaba la yerba, hacía hectáreas, hectáreas de yerba, entonces te daba para que vos plantes y para que vos mantengas limpio. En vez de pagarte para que vos limpies la chacra, entonces te da un pedazo para que vos plantes, entonces vos le mantenías limpia, sí o sí vos tenés que limpiar la chacra de él.

El caso de María Rosa (48 años, barrio Caballeriza) puede ubicarse en el marco de estos procesos de descomposición campesina, pero atravesada por una biografía particular. Hasta el año 2002 vivió en una chacra con su marido; ambos trabajaban simultáneamente en la cosecha de yerba y en la producción agrícola orientada al autoconsumo y venta de excedentes. Al quedar 
viuda, con sus cuatro hijos pequeños, decidió vender la chacra y trasladarse a un barrio periurbano de Oberá. Relata que el aislamiento y los problemas de acceso a transporte y escolarización de las zonas rurales incidieron en la decisión de su relocalización:

En la chacra, los caminos eran muy feos, estábamos muy lejos de la ruta, para salir a la ruta tenía que caminar kilómetros, cuando llovía era imposible y con los chicos se hacía difícil, para ir a la escuela era muy feo, muy lejos.

Sin embargo, como mencionamos antes, no todos los casos corresponden a situaciones campesinas o semicampesinas en crisis. Algunos sujetos residían en el campo pero no tenían producciones ni siquiera para el autoconsumo, se trataba de tareferos que circulaban por distintos períodos de tiempo entre las chacras de varios colonos y se instalaban (de modo muy precario) en las propiedades de los productores para los cuales trabajaban. Esta situación implicaba serias dificultades en el acceso a los servicios más básicos, como es el caso de Luis (35 años, Los Helechos), que hace diez años decidió trasladarse a un barrio en la periferia de Oberá, tras ver impedido el acceso de su familia a recursos como el agua potable y la electricidad, a pesar de residir en una zona con buen acceso a la ruta, lo que facilitaba al menos el acceso al transporte: "[...] por más que estábamos a la orilla del camino, la chacra pertenecía al dueño de la tierra y no autorizaba a bajar la luz, y no teníamos agua tampoco".

De este modo, las restricciones en el acceso a los servicios básicos (electricidad, agua potable), al transporte y a la escuela, junto a la expectativa de resolverlas en la ciudad, aparecen constantemente como estímulos a la relocalización de las familias; a estos motivos se suman muchas veces hitos familiares, como la muerte de un pariente, la separación de un cónyuge o los proyectos de escolarización de los hijos con la esperanza de desvinculación del trabajo agrícola en un futuro más o menos cercano.

Como se verá a continuación, tal como surge de la propia evaluación de los sujetos entrevistados, la escasa o a veces nula presencia de agentes y dispositivos estatales en el medio rural indirectamente estimula la relocalización, ante el despliegue de políticas de protección social en zonas urbanas y las mayores posibilidades de acceso, ya sea más rápido o en forma más directa.

\section{"Ahora finaliza la cosecha y quedamos en el olvido, el patrón te larga y arreglate como podés"}

Para estos trabajadores y sus hogares, ahora residentes en áreas urbanas, el nuevo escenario laboral tiene a la continuidad de la tarefa como ocupación principal y a veces casi exclusiva - aunque organizada sobre condiciones distintas a las de la etapa anterior- y la combinación con un conjunto li- 
mitado e igualmente inestable de otras ocupaciones. En el nuevo escenario periurbano, la dimensión laboral atraviesa la vida cotidiana de sus habitantes a través de las redes conformadas por vínculos entre vecinos y parientes.

De cualquier manera, la relocalización de la mano de obra en barrios periurbanos no elimina, sin embargo, la movilidad espacial vinculada a la mencionada continuidad en el trabajo con la yerba, que a su vez se sostiene por las escasas oportunidades para insertarse en ocupaciones urbanas.

En este contexto, el hecho de que la extensión máxima de la tarefa se haya reducido a seis meses (antes podía extenderse hasta nueve meses) consolida una situación de extrema precariedad laboral y de riesgo para la sobrevivencia de esta población. Esto ocurre aun cuando el nivel de formalización en el trabajo ha venido creciendo y con ello el acceso a algunos beneficios de la protección y la seguridad social. "La realidad de los tareferos es terminó la cosecha y arreglate como podés, el patrón si es posible ni te saluda y te larga" (Ramón, 36 años, barrio La Cantera).

Con relación a los trabajos temporales que los sujetos pueden activar entre cosechas, se pueden mencionar la construcción, la fabricación de ladrillos y otros trabajos agrícolas como la cosecha de té. Sin embargo, se trata de trabajos esporádicos con remuneraciones muy bajas y que no siempre se encuentran disponibles en los períodos entre cosechas.

Hay gente que la pasa mal porque no tienen ningún trabajo, nada de nada; se quedan meses esperando y a veces empiezan a vender las cosas que tienen en la casa y lo único que les queda es esperar la próxima cosecha (Luis, 35 años, Los Helechos).

Además, el bajo nivel de escolarización (las generaciones más jóvenes suelen tener solo el ciclo primario completo, mientras que sus padres en muchos casos no lo han completado e incluso persisten casos de analfabetismo) funciona como una barrera en el acceso a otros trabajos disponibles en los espacios urbanos, como por ejemplo el empleo en comercio.

El barrio se constituye en un escenario estratégico para la consolidación de dos figuras claves para el funcionamiento de estos mercados de trabajo: el contratista y el capataz. El primero funciona como interlocutor para los patrones - incluyendo productores y empresas - para el reclutamiento, el transporte y la gestión administrativa de los trabajadores en los yerbales; es visto por los trabajadores como una forma de desvinculación del patrón hacia los tareferos y generalmente es una figura con connotaciones negativas.

Antes no había el tema de contratistas, antes no existía eso... Había un patrón que hacía todo y ahora hay muchos contratistas y los patrones se lavan las manos, como dicen... Perjudicaron a los trabajadores y ellos se beneficiaron porque se llenaron de plata (Carlos, 29 años, Villa Bonita). 
Nosotros lo que necesitamos es terminar con el contratista, porque se lleva la mejor tajada, que el patrón se haga responsable, que vuelva a ser esa vida de antes (Carlos, 53 años, Los Helechos).

En cambio, el capataz, si bien trabaja para un contratista, es una figura más cercana al trabajador y puede residir en los mismos barrios de cosecheros y manejar una amplia red de contactos amicales, vecinales y parentales, además de permanecer controlando la labor de cosecha. Es una figura más ambigua, es el que "controla" a los trabajadores y su remuneración depende de eso, pero, a la vez, un "buen capataz" es el que "sabe cuidar a la gente". En relación con esto, Simeón (65 años) relata la experiencia de haber oficiado como capataz:

Porque uno tiene que saber, uno que va a tener cuadrilla... Porque yo sé, uno tiene que saber cuidar a la gente y por supuesto que hay que cuidar a la yerba para el colono, porque si el colono deja fundir la yerba, no va a haber más tarefa. Y tenemos que hacer con cuidado todo, ser honesto(s), digamos.

De este modo, el vínculo del capataz con los trabajadores es a la vez un vínculo cotidiano de amistad, vecindad o parentesco sobre el que se construye la relación de trabajo. Los cosecheros pueden trabajar con el mismo contratista por períodos prolongados:

No es difícil conseguir para ir a tarefear, porque yo voy hace años, entonces yo digo "Bueno, yo puedo ir a tarefear con vos (se refiere al capataz)". "Sí, vamos", dice. Y listo, viste, ya ellos me conocen, entonces es más fácil. O ya me preguntan antes de empezar la tarefa, “¿Vamos a ir este año?", dice. "No sé, vamos a ver", le digo, y así, viste (Emilia, 27 años, Villa Bonita).

Sin embargo, la cercanía que existe en el vínculo entre capataces y trabajadores no está exenta de conflictos que pueden llevar a la interrupción de la relación laboral; en los casos de vínculos prolongados, se trata generalmente de conflictos personales que llevan a que el trabajador cambie de intermediario. Elisa (48 años, Los Helechos) relata: "[...] con mi marido fui por diez años con el mismo capataz, yo me hice muy amiga de la mujer [...] pero un día se peleó con mi marido muy feo y de ahí ya no fuimos más".

La residencia en los barrios influyó también, para la cosecha, en la decisión de optar por trasladarse diariamente entre la vivienda y los yerbales antes que la modalidad de campamento, que implica permanecer en el lugar de trabajo durante una semana o incluso más para luego regresar al domicilio, y después repetir esta circulación hasta la finalización de la campaña. Algunos de los motivos son económicos, como los referidos a los mayores costos de los alimentos que les proveen, que luego son descontados del salario, pero también tienen que ver con un mayor margen de autonomía, en el caso de la 
modalidad de traslado diario, y con la posibilidad de compartir más tiempo con la familia.

Qué sé yo, lo que más me gusta es que puedo compartir con mi familia más tiempo, y que depende más de mí... No te están obligando a tal hora tenés que estar acá... Siempre me gustó porque es un trabajo casi libre, te tomás tu tiempo al ritmo que quiera(s) (Emilia, 27 años, Villa Bonita).

En los últimos cinco años, Emilia decidió dejar de trabajar en la modalidad de campamento:

Es más difícil porque vos no ves a tus hijos todos los días, digamos, vos te vas lunes o domingo y volvés recién el viernes y durante todo ese tiempo uno no ve y es difícil. Para mí fue bastante difícil eso (Emilia, 27 años, Villa Bonita).

Con respecto a la participación de la mujer en la tarefa, ha estado invisibilizada en la categoría de "ayudas familiares" (Haugg, 2018), que concebía como cosechero al varón de la familia, principalmente por su vínculo con el contratista. Aun cuando a partir de los años noventa se reconoce a la mujer como asalariada de la cosecha de yerba, persisten significaciones que siguen ubicando a las tareferas en un lugar de invisibilización, tan es así, que algunas mujeres que cosechan junto con sus maridos se autoperciben como "ayudantes del varón", situación que no sucede en el caso de aquellas mujeres que ocupan el rol de jefas de familia, que se autoperciben como tareferas. Las tareas que frecuentemente realizan las mujeres en la tarefa son el corte y el quiebre de la yerba; la carga de los raídos en los camiones en general es realizada por varones, aunque algunas mujeres relatan haber realizado cargas en alguna oportunidad.

\section{"Acá la mayoría son conocidos o parientes, hasta si alguno se queda sin trabajo se va a la casa del pariente"}

Vivir en los barrios implica la posibilidad de acceso a recursos económicos y a infraestructura, a beneficiarse, y también involucrarse, de acciones de solidaridad y afectivas para el cuidado de las familias y a la atención de situaciones particularmente críticas, muchas de ellas derivadas de la falta de trabajo.

En este sentido, la instalación en los barrios debe comprenderse principalmente en relación con la existencia de redes de contención parental-vecinal y con el aprovechamiento de políticas públicas de distinto tipo; los lazos comunitarios y el Estado en sus distintos niveles aparecen compensando las privaciones derivadas de las dificultades en el acceso al trabajo

La vivienda es un aspecto crítico para resolver, incluso previo al acceso a un trabajo. El terreno y los materiales para la construcción de la vivienda 
son gestionados, al menos parcialmente, por las políticas municipales. Al mismo tiempo, se trata de una población que percibe políticas de transferencia monetaria (políticas generalizadas a nivel nacional) como la asignación universal por hijo (AUH), ${ }^{11}$ pensión por ser madre de siete hijos, jubilaciones no contributivas (dirigidas a personas que trabajaron por fuera del régimen laboral registrado) y pensiones por discapacidad. Dentro de las políticas de protección social vinculadas específicamente a la población cosechera, en el nivel provincial y municipal cabe mencionar los "bolsones de alimentos" y a nivel nacional el Programa Intercosecha ${ }^{12}$ (antes conocido como Interzafra), consistente en un subsidio que perciben los tareferos en los intervalos de tiempo entre cosechas.

Estela (42 años, barrio Escondido), hija de tareferos, no completó la escuela porque, desde chica, tenía que acompañar a su padre a los campamentos para tarefear; luego de separarse de su primer esposo, se mudó para el barrio con sus seis hijos y la red de contención familiar le posibilitó la instalación: "Me vine primero a vivir en la casa de mi hermana, comíamos todos juntos [...]. Después conocí y tuve a mi segundo marido, con él hicimos nuestra casa, el intendente nos dio un terreno".

Miguel (35 años, barrio San Miguel) relata que un familiar directo

estaba metido con el ministerio y él consiguió una casita de cuatro por seis, de media agua... y me consiguió una para mí... Te pagaban los materiales o venían y te ponían la base, pagábamos nosotros para hacer la casita... y ahí quedabas.

En otros casos, los terrenos fueron cedidos directamente por el gobierno municipal:

No es muy antiguo el barrio, hace unos años vivíamos más abajo, al otro lado del camino, y entonces el intendente compró estos terrenos y nos movieron a todos acá (Estela, 42 años, barrio Escondido).

En algunos casos, varias familias instalaron sus viviendas en un mismo terreno y comparten algunos gastos. Luis (35 años) vive con su mujer y comparte el terreno con uno de sus tíos, con el que dividen los gastos del servicio de energía eléctrica y reparten la mercadería que reciben en los bolsones de

11 La AUH es una política de transferencia condicionada de ingresos que se implementa desde 2009 y se otorga mensualmente a personas desocupadas, empleados no registrados o que ganan menos del salario mínimo legal, se calcula por cada hijo menor de 18 años e hijo con discapacidad. Desde 2011 se integró con la asignación universal por embarazo para mujeres a partir de las 12 semanas de gestación.

12 El Programa Intercosecha está dirigido a trabajadores agrícolas registrados y brinda una ayuda económica no remunerativa durante el período de receso de la actividad por un máximo de cuatro meses (equivalente aproximadamente a la mitad del salario mínimo legal). 
alimentos que les provee el Estado provincial. Luis expresa que los parientes del barrio funcionan como una red de contención en las épocas en que no hay cosecha de yerba: "El tema es que acá la mayoría son conocidos, son parientes... entonces si alguno se queda sin trabajo se va a la casa del pariente". Una estrategia similar despliega Pedro (44 años, barrio San Miguel), quien vive con seis de sus ocho hijos y está construyendo en el fondo de su terreno la casa de su hija, que a su vez vive con su esposo e hijo.

Excepto la tarefa, el resto de los trabajos no agrícolas se consiguen a partir de la circulación de información entre vecinos y parientes. Luis (35 años, Los Helechos) sostiene:

Yo tengo muchos conocidos, me vienen a buscar, siempre vienen a preguntarme. Pero también a veces viene alguien y me dice, "Necesito cinco personas, ¿a quién me recomendás?". Y yo le digo: "Andá a la casa de fulano". "Necesito arreglar una pared", "Fulano sabe de esto".

También las redes parentales y vecinales se activan frente a las tensiones entre el trabajo productivo y reproductivo de las mujeres cosecheras. Emilia (27 años, Villa Bonita) es tarefera, nieta e hija de tareferos, jefa de familia. Tiene seis hijos y relata cómo entre las vecinas comparten el trabajo de cuidados y tareas domésticas:

[...] a mi vecina a veces le paso plata para que me cuide a los nenes y otras veces no [...]. Es mi amiga, a veces no me cobra nada, me dice "Tráelos, traé la carne, las cosas para cocinar y listo, yo te cuido" [...]. Cuando llega el fin de semana yo lavo la ropa, todas las cosas, después yo la ayudo a ella (refiriéndose a la vecina), lavo la ropa de ella con la mía.

Con relación a los bolsones de alimentos, es frecuente que algunos pobladores deban trasladarse a la ciudad de Posadas (capital de la provincia de Misiones) para acceder a ellos y luego hacerse cargo de su distribución en los barrios. El traslado a la capital también se vincula con trámites administrativos para acceder a otros beneficios, como el mencionado Programa Intercosecha. Los costos de estos traslados quedan a cargo de los trabajadores, quienes no siempre pueden afrontarlos, lo que se traduce, en algunos casos, en la imposibilidad de acceder a los beneficios.

La incompatibilidad entre la percepción de políticas sociales y el registro laboral lleva a que sean las mujeres las más afectadas, al ser titulares de algunos de los beneficios; por ejemplo, resulta incompatible percibir la AUH durante aquellos meses en los que están registradas y, en el proceso para volver a percibirla, pueden pasar varios meses sin recibir ningún ingreso.

De este modo, algunas mujeres "deciden" trabajar en negro para no perder la regularidad del ingreso vía política social, obligadas de este modo a renunciar a derechos elementales como la jubilación y la cobertura de salud. 
Esta situación lleva incluso a que algunas perciban el registro laboral como algo problemático. Tal es el caso de Elsa (48 años, Los Helechos), que expresa: "y antes era más fácil poder trabajar para las mujeres, porque no te exigían estar fichada (se refiere al registro laboral)... Si ficho me cortan mi pensión”.

\section{Conclusiones}

La relocalización en barrios urbanos por parte de trabajadores y familias rurales remite a un proceso de construcción de una nueva territorialidad, en la cual el trabajo comparte su función organizadora de esos espacios junto a otras prácticas vinculadas más bien a procesos de reproducción social en un sentido amplio.

En principio, la restructuración productiva en la actividad yerbatera, que tiende a profundizar las condiciones de temporalidad del empleo agrario y a privilegiar la tercerización como forma de contratación casi exclusiva de trabajadores, se da en un contexto de disminución general de los requerimientos de trabajo, concentrándolos aún más en momentos puntuales del ciclo productivo y generando con ello excedentes de población rural activa. Mientras que para la agroindustria yerbatera, los productores y los contratistas los barrios de trabajadores se constituyen en reservorio de mano de obra barata siempre disponible, para los trabajadores y trabajadoras y sus familias constituyen un tejido de relaciones sociales que posibilitan la reproducción social cotidiana simbólica y material.

Durante las etapas previas a la restructuración que se aceleró desde los años noventa, los testimonios que reconocen la existencia de "más trabajo" refieren fundamentalmente a las posibilidades concretas de estar ocupado durante más tiempo a lo largo del año, en el mismo cultivo de la yerba y en otros cultivos que les permitían complementar el anterior. Este mayor nivel de ocupación de alguna manera los lleva a relativizar actualmente la precariedad de esas ocupaciones.

El crecimiento de la intermediación laboral (con el contratista y el capataz como figuras claves) y la consecuente baja presencia de los patrones son vistos como expresiones asociadas a esas situaciones de falta de trabajo, en algunos casos ocasionándolas y en otros precarizando aún más las inserciones ocupacionales. Sin embargo, principalmente con el capataz se busca mantener un vínculo continuo y estratégico para garantizar disponibilidad de trabajadores para las empresas y niveles de ocupación lo más prolongados posible en el tiempo para los trabajadores.

La movilidad hacia los centros urbanos no se funda en posibilidades concretas, ni siquiera, muchas veces, en expectativas de una potencial inserción laboral en actividades urbanas. Una situación generalizada de changas, con remuneraciones bajas y sin ningún tipo de protección social es típica de 
la mayoría de las ocupaciones urbanas para esta población; a veces, el bajo nivel educativo alcanzado limita el acceso a eventuales mejores trabajos, pero en estas condiciones de vida la deserción termina siendo una conducta frecuente.

En la decisión y en el proceso mismo de relocalización en barrios periurbanos y en pequeñas localidades urbanas se hace evidente la necesidad de disponer de redes conformadas a través de lazos de vecindad, parentesco y sociabilidad en general, para enfrentar las nuevas condiciones de acceso al trabajo y para sostener la reproducción social.

En este contexto, el Estado en sus distintos niveles y a partir de diferentes intervenciones y políticas — subsidios, transferencias directas de ingresos, alimentos - se convierte en el instrumento clave para la reproducción de esta población, principalmente mediante la transferencia de recursos, en general magros pero estables, especialmente durante los períodos de escasez de trabajo. Así, la ciudad, por medio del Estado, cumple una función que antes le correspondía a la propia actividad agrícola, a partir de la presencia de oportunidades de trabajo más extendidas en el tiempo y en muchos casos con la autoproducción de alimentos.

En resumen, una nueva espacialidad de los trabajadores agrícolas y sus familias no implica necesariamente una ruptura con su pasado laboral: incluye el mantenimiento de prácticas laborales previas y la complementariedad con otras nuevas, pero también el necesario desarrollo de estrategias específicas para acceder a los recursos que les garanticen su supervivencia. Esto último resulta clave tratándose de sujetos que atraviesan una doble exclusión: la precariedad de las inserciones laborales en las áreas rurales y las críticas condiciones de vida en las ciudades que constituyen su nuevo entorno de sociabilidad y de vida.

\section{Referencias bibliográficas}

AACREA (Asociación Argentina de Consorcios Regionales de Experimentación Agrícola) (2003). Yerba mate. Buenos Aires: Agroalimentos Argentinos, pp. 141-146.

Albertí, A. (2018). De "ayudas merecidas y no merecidas". Las políticas sociales de transferencia monetaria en la zona rural del nordeste de Misiones, Argentina. Cuadernos de la Facultad de Humanidades y Ciencias Sociales. 54, pp. 115-138.

Albertí, A. y E. Mingo (2019). Tensiones y ambigüedades de las políticas sociales en el mundo del trabajo agrícola. La asignación universal por hijo en Tucumán y Misiones, Argentina. Cuadernos de antropología Social, 49, pp. 75-90. 
Aparicio, S.; D. Re y S. Pereyra (2016). Misiones: migración y yerba mate. En: S. Aparicio y R. Benencia (comps.). De migrantes y asentados: trabajo estacional en el agro argentino. Buenos Aires: CICCUS, pp. 99-114.

Barrón, A. (1999). Las migraciones en los mercados de trabajo de cultivos intensivos en fuerza de trabajo: un estudio comparativo. En: H. de Grammont, M. A. Gómez Cruz, H. González y R. Schwentesius (comps.). Agricultura de exportación en tiempos de globalización. México: RISHORT, CIESTAAM, UNAM, CIESAS.

Bartolomé, L. (1975). Colonos, plantadores y agroindustrias. La explotación agrícola familiar en el sudeste de Misiones. Desarrollo Económico, 15(58), pp. 239-264.

Bolsi, A. (1982). La yerba mate y la ocupación del espacio misionero. Boletín de Estudios Geográficos, 21(80), pp. 7-65.

Colcombet, L. (2010). La demanda de mano de obra en yerba mate, provincia de Misiones. En: G. Neiman (dir.). Estudio de la demanda de mano de obra en el agro argentino. Buenos Aires: CICCUS, pp. 133-148.

Crovetto, M. M. (2011). Movilidad espacial, ocupación y empleo en el Valle Inferior del Río Chubut. Trabajo y Sociedad, 17, pp. 363-380.

Fabio, J. F. (2009). Conformación social y local de la temporalidad. Los mercados de trabajo en las producciones de yerba mate y uvas. 9. ${ }^{\circ}$ Congreso Nacional de Estudios del Trabajo, Asociación Nacional de Especialistas en Estudios del Trabajo (ASET), Buenos Aires.

Flood, C.; M. Caracciolo; S. Baudron; M. B. Dourron; A. Paura y N. Fuentes (1972). Estudio de la mano de obra transitoria de la provincia de Misiones. Buenos Aires: Ministerio de Agricultura y Ganadería de la Nación.

Forni, F. H. (1965). Encuesta socio-rural de la provincia de Misiones. Primera parte: Situación social de la población rural. Buenos Aires: Instituto Nacional de Tecnología Agropecuaria (INTA).

Gallero, M. C. (2019). Cambios y permanencias en la producción de yerba mate: Un estudio desde la historia ambiental en Misiones (Argentina). Historia Ambiental, Latinoamericana y Caribeña, 9(1), pp. 223-257.

Gómez Lende, S. (2016). Agricultura, agroindustria y territorio: crisis y reestructuración del circuito de la yerba-mate en la provincia de Misiones (Argentina) 1990-2014. Cuadernos de Geografia: Revista Colombiana de Geografía, 25(1), pp. 45-63. 
Gomez, S. y E. Klein (1993). Los pobres del campo. El trabajo eventual. Santiago de Chile: FLACSO/PREALC.

Graziano da Silva, J. (1982). A modernização dolorosa: estrutura agrária, fronteira agrícola e trabalhadores rurais no Brasil. Rio de Janeiro: Zahar.

Gorenstein, S.; M. Schorr y G. Soler (2011). Dilemas estructurales del norte argentino. Un enfoque estilizado de tres complejos agroindustriales de la región. Estudios Urbanos e Regionais, 13(1), pp. 27-50.

Gortari, J. (2016). Economía regional yerbatera: acumulación de capital con exclusión social. En: J. Gortari, V. Rosenfeld y A. Oviedo (comps.). Dinámica agraria y políticas públicas. Desigualdades sociales y regionales. Posadas, Misiones: Editorial Universitaria, pp. 63-86.

Gortari, J.; D. Re y M. L. Roa (comps.) (2017). Tareferos: vida y trabajo en los yerbales. Misiones: Edunam. Editorial Universitaria de la Universidad Nacional de Misiones.

Gortari, J. y A. Sena (2018). Economía regional yerbatera: logros y limitaciones en 15 años de funcionamiento del Instituto Nacional de la Yerba Mate - INYM. Posadas: Editorial Universitaria de la Universidad Nacional de Misiones.

Haesbaert, R. (2009). Dilema de conceitos: espacio-territorio e contencão territorial. En M. A. Saquet y E. S. Sposito (orgs.). Territorios e territorialidades: teorías, procesos y conflictos. Sao Paulo: Editorial Expressão Popular, pp. 95-120.

Haesbaert, R. (2013). Del mito de la desterritorialización a la multiterritorialidad. Cultura y representaciones sociales, 8(15), pp. 9-42.

Haugg, D. (2018). “Ahora trabajo, antes solo ayudaba": algunas aproximaciones históricas al trabajo femenino en los yerbales. Oberá, Misiones, Argentina. En: F. Jaume (comp.). Luchas por la hegemonía. Historia y etnografía en la Provincia de Misiones, Argentina. Posadas: EDUNAM, pp. 331-370.

Ibarguren, M. (2017). Contratismo rural en la actividad yerbatera: ¿hombres de paja o empresas de servicios? En: J. Gortari, D. Re y M. L. Roa (comps.). Tareferos: vida y trabajo en los yerbales. Posadas: Edunam, pp. 303-328.

INDEC (Instituto Nacional de Estadística y Censos) (2002). Censo Nacional Agropecuario. Buenos Aires: INDEC.

INDEC (Instituto Nacional de Estadística y Censos) (2018). Censo Nacional Agropecuario, resultados provisionales. Buenos Aires: INDEC. 
INDEC (Instituto Nacional de Estadística y Censos) (2010). Censo Nacional de Población y Viviendas. Buenos Aires: INDEC.

Lara, S. M. (1998). Nuevas experiencias productivas y nuevas formas de organización flexible del trabajo en la agricultura mexicana. México: Procuraduría Agraria-Juan Pablos Editor.

Lara, S. M. (2012). Los territorios migratorios como espacios de articulación de migraciones nacionales e internacionales. Cuatro casos del contexto mexicano. Política y Sociedad, 49(1), pp. 89-102.

Lindón, A. (2002a). La construcción social del territorio y los modos de vida en la periferia metropolitana. Territorios, 7, pp. 27-41.

Lindón. A. (2002b). Trabajo, espacios de vida y cotidianidad. La periferia oriental de la ciudad de México. Scripta Nova, VI(119). Disponible en: $<$ http:// www.ub.es/geocrit/sn/sn119-56.htm> [acceso 6/12/2020].

Lindón, A. (2015). Del cronotopo fundacional a la construcción socio-espacial del territorio vallechalquense. En: A. Lindón y C. Mendoza (coords.). La periferia metropolitana: entre la ciudad prometida y un lugar para habitar en la ciudad de México. Barcelona: Gedisa, pp. 115-150.

Ministerio de Trabajo, Empleo y Seguridad Social de la Nación (2014). Encuesta de asalariados agrarios sobre protección social, empleo y condiciones de trabajo. Buenos Aires: Ministerio de Trabajo, Empleo y Seguridad Social de la Nación.

Moreno, M. S. (2019). Territorialidad inmigratoria de trabajadores bolivianos en Mendoza (Argentina). Aproximación etnográfica a partir de tres casos de estudio emplazados en espacios rurales. Territorios, 41, pp. 23-43.

Narotzky, S. (2004). Antropología económica: nuevas tendencias. Barcelona: Melusina.

Neiman, G. y G. Quaranta (2000). Reestructuración de la producción y flexibilidad funcional del trabajo agrícola en la Argentina. Revista Latinoamericana de Estudios del Trabajo, 12(7), pp. 45-70.

Neiman, G. (2010). Los estudios sobre el trabajo agrario en la última década: una revisión para el caso argentino. Mundo Agrario, 10(20), pp. 112-131.

Pérez Martínez, M. (2016). Las territorialidades urbano-rurales contemporáneas: un debate epistémico y metodológico para su abordaje. Bitácora Urbano Territorial, 26(2), pp. 103-112. 
Piñeiro, D. (1999). Los trabajadores rurales en tiempos de flexibilidad. El caso de Uruguay. Revista de Ciencias Sociales, 16, pp. 99-113.

Quaranta, G. (2010). Estructura ocupacional, características de la demanda y perfil de los trabajadores en el agro argentino a principios de la década actual. En: G. Neiman (dir.). Estudio de la demanda de mano de obra en el agro argentino. Buenos Aires: CICCUS, pp. 13-50.

Radonich, M. (2001). Asentamientos de trabajadores migrantes y redefinición de estrategias sociolaborales en el Alto Valle del río Negro y del Neuquén. 5. Congreso Nacional de Estudios del Trabajo, ASET, Buenos Aires, Argentina, agosto.

Rau, V. (2014a). Cosechando yerba mate: estructuras sociales de un mercado laboral agrario en el Nordeste Argentino. Buenos Aires: CICCUS.

Rau, V. (2014b). Un resurgimiento de la intermediación laboral. Los contratistas de mano de obra en el mercado de trabajo agrícola yerbatero. En: R. Benencia y S. Aparicio. Nuevas formas de contratación en el trabajo agrario. Buenos Aires: CICCUS, pp. 71-93.

Rodríguez, L. (2018). La agroindustria yerbatera argentina: industria y acción colectiva en la frontera, Misiones (1991-2013).H-industri@, Revista de Historia de la Industria, 23(12), pp. 55-69.

Rofman, A. (2001). Estructuras regionales y sistemas productivos. Buenos Aires: Aique.

Saquet, M. (2015). Por una geografía de las territorialidades y las temporalidades: una concepción multidimensional orientada a la cooperación y el desarrollo territorial [en línea]. La Plata: Facultad de Humanidades y Ciencias de la Educación, Universidad Nacional de La Plata. Disponible en: $<$ http://www.memoria.fahce.unlp.edu.ar/libros/pm.268/pm.268.pdf $>$ [acceso $10 / 12 / 2020]$.

Schiavoni, G. (2008). Repensar la reproducción. Del campesinado a la agricultura familiar. En: G. Schiavoni (comp.). Campesinos y agricultores familiares. La cuestión agraria en Misiones a fines del siglo XX. Buenos Aires: CICCUS, pp. 13-31.

Sena, A. (2017). El circuito productivo de la yerba mate. Transformaciones, cambios y continuidades en el periodo post convertibilidad (2002-2016). Tesis de Maestría en Desarrollo Rural. Posadas: Universidad Nacional 
de Misiones. Disponible en: <https://rid.unam.edu.ar/bitstream/handle/20.500.12219/1171/SENA-Anibal-2017-Circuito-productivo-Yerba-MateTesis-MDR.pdf?sequence=1\&isAllowed=y $>$ [acceso 3/11/2020].

Slutzky, D (2011). Estructura social agraria y agroindustrial del nordeste de la Argentina: desde la incorporación a la economía nacional al actual subdesarrollo concentrador y excluyente. Buenos Aires: Instituto Argentino para el Desarrollo Económico (IADE).

Tarrius, A. (2000). Leer, describir, interpretar. Las circulaciones migratorias: conveniencia de la noción de "territorio circulatorio". Los nuevos hábitos de la identidad. Relaciones. Estudios de historia y sociedad, 83, pp. 39-66

Trpin, V (2020). Somos libres, cuando queremos nos vamos. Sindicalismo, migrantes chileno/as y norteños/as en la fruticultura del norte de la Patagonia. Comahue: Universidad de los Lagos.

Tubío, M. (2001). El impacto de las transformaciones agrarias sobre el empleo rural en el Uruguay. Buenos Aires: CLACSO.

Wikipedia (2007). Municipio Oberá en departamento Oberá [mapa]. Wikipedia [en línea]. Disponible en: <https://es.wikipedia.org/wiki/Ober\%C3\%A1\#/ media/Archivo:Municipio_Ober\%C3\%A1_en_departamento_ Ober\%C3\%A1.png> [acceso 26/05/2021].

\section{Contribución de autoría}

Este trabajo fue realizado en partes iguales por Guillermo Neiman y Alfonsina Verónica Albertí. ${ }^{13}$

13 Los autores agradecen los comentarios de dos revisores anónimos, que contribuyeron a mejorar este artículo; a Silvia Bardomás y Gabriel Bober, que realizaron entrevistas; y a Francisco Fabio, por su colaboración en el trabajo de campo. 